\title{
SOME CHARACTERIZATIONS OF STEIN MANIFOLD THROUGH THE NOTION OF LOCALLY REGULAR BOUNDARY POINTS
}

\author{
By JOJI KaJIWARA
}

Dedicated to Professor K. Kunugi on his sixtieth birthday

\section{Introduction.}

The main purpose of the present paper is to investigate the intersection of a Cousin-I domain and a domain of holomorphy. Oka [14] proved that a domain of holomorphy in $C^{n}$ is a Cousin-I domain, that is, a domain in which any additive Cousin's distribution has a solution. On the other hand, a Cousin-I domain in $C^{2}$ is a domain of holomorphy from Cartan [5] and Behnke-Stein [2]. Therefore a domain in $C^{2}$ is a Cousin-I domain if and only if it is a domain of holomorphy. Cartan [6] proved that $E=\left\{\left(z_{1}, z_{2}, z_{3}\right) ;\left|z_{1}\right|<1,\left|z_{2}\right|<1,\left|z_{3}\right|<1\right\}-\{(0,0,0)\}$ is not a domain of holomorphy but a Cousin-I domain. For any domain of holomorphy $D$ in $C^{3}, E \cap D$ is a Cousin-I open set. Making use of the results of Scheja [16] and Andreotti-Grauert [1] concerning the prolongation of cohomology classes, we can construct systematically other Cousin-I domains in $C^{n}$ which are not domains of holomorphy for $n \geqq 3$. For $G=\left\{\left(z_{1}, z_{2}, z_{3}\right) ;\left|z_{1}\right|<1,\left|z_{2}\right|<1,\left|z_{3}\right|<1\right\}-\left\{\left(z_{1}, z_{2}, z_{3}\right) ; z_{1}=z_{2}\right.$ $\left.=0,\left|z_{3}\right| \leqq 1 / 2\right\}$, there holds $H^{1}(G, \mathfrak{D})=0$ from Scheja [16] where $\mathfrak{D}$ is the sheaf of all germs of holomorphic functions. Therefore $G$ is not a domain of holomorphy but a Cousin-I domain. But $G$ has a different property from $E$. The intersection $G \cap Z=\left[\left\{\left(z_{1}, z_{2}\right) ;\left|z_{1}\right|<1 / 2,\left|z_{2}\right|<1 / 2\right\}-\{(0,0)\}\right] \times\left\{z_{3} ;\left|z_{3}\right|<1 / 2\right\}$ of $G$ and a tridisc $Z=\left\{\left(z_{1}, z_{2}, z_{3}\right) ;\left|z_{1}\right|<1 / 2,\left|z_{2}\right|<1 / 2,\left|z_{3}\right|<1 / 2\right\}$ is not a Cousin-I domain as the first component of the above direct product is not a Cousin-I domain from the results of Cartan [5] and Behnke-Stein [2].

A domain in $C^{n}$, which is a direct product $K_{1} \times K_{2} \times \cdots \times K_{n}$ of relatively compact subdomains $K_{2}$ of a complex plane, is called a polycylinder hereafter. An open set $G$ in $C^{n}$ is called regular if $G \cap\left(K_{1} \times K_{2} \times \cdots \times K_{n}\right)$ is a Cousin-I open set for any polycylinder $K_{1} \times K_{2} \times \cdots \times K_{n}$ in $C^{n}$. From the previous paper [12] of the author $G$ is a Cousin-I open set. Cartan's example $E$ is a regular domain in $C^{3}$ but the above example $G$ is not a regular domain. We say that a domain $G$ in $C^{n}$ is exhausted by regular domains if there exists a sequence $\left\{G_{p} ; p=1,2,3, \cdots\right\}$ of regular domains $G_{p}$ such that $G_{p} \Subset G_{p+1}(p=1,2,3, \cdots)$ and $G=\cup_{p=1}^{\infty} G_{p}$. From the previous paper [12] of the author $G$ is a Cousin-I domain as it is a limit of mono-

Received May 19, 1964. 
tonously increasing sequence of Cousin-I domains $G_{p}$. Moreover we shall prove that a domain in $C^{n}$ is a domain of holomorphy if and only if it can be exhausted by regular domains. This is a characterization of a domain of holomorphy by means of Cousin-I problems. This means that a regular domain in $C^{n}$, which is not a domain of holomorphy, is isolated in the set of regular domains in some sense.

We shall define a continuous boundary point of an open set in $C^{n}$ in such a way that a smooth boundary point of an open set in $C^{n}$ in the usual sense is a continuous boundary point. An open set $G$ in $C^{n}$ is called locally regular at a boundary point $z^{0}$ of $G$ if there exists an open neighbourhood $U$ of $z^{0}$ such that $G \cap U$ is regular. An open set is called locally regular if it is locally regular at each of its boundary points. We shall prove that a domain is pseudoconvex at its continuous boundary point $z^{0}$ if and only if $G$ is locally regular at $z^{0}$. Hence from the affirmative solution of the Levi problem due to Bremermann [4], Norguet [13] and Oka [15] a domain with a continuous boundary is a domain of holomorphy if and only if it is locally regular. This is a characterization of a domain of holomorphy with a continuous boundary by means of Cousin-I problems. Making use of Docquier-Grauert [8] we shall extend this fact to a domain in a Stein manifold.

\section{$\S 1$. Domain exhausted by regular domains.}

Lemma 1. Let $G$ be a regular domain in $C^{n}$. Then $D=G \cap\left\{z=\left(z_{1}, z_{2}, \cdots, z_{n}\right)\right.$; $\left.z_{j} \in K_{j}\left(j=s_{1}, s_{2}, \cdots, s_{r}\right)\right\}$ is a Cousin-I open set for any $1 \leqq s_{1}<s_{2}<\cdots<s_{r} \leqq n$ and for any domains $K_{J}$ in a complex plane $\left(j=s_{1}, s_{2}, \cdots, s_{r}\right)$. Especially $G$ itself is a Cousin-I domain.

Proof. We put $K_{j}^{p}=\left\{z_{j} ;\left|z_{j}\right|<p\right\}$ for $j \notin\left\{s_{1}, s_{2}, \cdots, s_{r}\right\}$ and $K_{j}^{p}=K_{j} \cap\left\{z_{j} ;\left|z_{j}\right|<p\right\}$ for $j \in\left\{s_{1}, s_{2}, \cdots, s_{r}\right\}$. Then $D_{p}=G \cap\left(K_{1}^{p} \times K_{2}^{p} \times \cdots \times K_{n}^{p}\right)$ is a Cousin-I open set for each $p$ as $G$ is a regular domain. Since $D$ is the limit of a monotonously increasing sequence of Cousin-I open sets $D_{p}, D$ is a Cousin-I open set from the previous paper [12] of the author. In the same way we can prove that $G$ itself is a CousinI domain.

The proof of the following Lemmas 2 and 3 is similar to the method of Hitotumatu [10].

Lemma 2. Let $G$ be a Cousin-I domain in $C^{n}$ and $H$ be an (n-1)-dimensional analytic plane in $C^{n}$. Then the inclusion map $G \cap H \rightarrow G$ induces naturally $a$ homomorphism of $H^{0}(G, \mathfrak{Q})$ onto $H^{0}(G \cap H, \mathfrak{Q})$.

Proof. Without loss of generality we may suppose that $H=\left\{(z, w)=\left(z_{1}, z_{2}, \cdots\right.\right.$, $\left.\left.z_{n-1}, w\right) ; w=0\right\}$. Let $u(z)$ be a holomorphic function in $G \cap H$. If $x^{0}=\left(z^{0}, 0\right)=\left(z_{1}^{0}, z_{2}^{0}\right.$, $\left.\cdots, z_{n-1}^{0}, 0\right)$ is a point of $G \cap H$, there exists a neighbourhood $U\left(x^{0}\right)=\left\{(z, w) ;\left|z_{j}-z_{j}^{0}\right|\right.$ $<\varepsilon,|w|<\varepsilon(j=1,2, \cdots, n-1)\}$ of $x^{0}$ in $G$. If $x^{0}$ is a point of $G-G \cap H$, we put $U\left(x^{0}\right)=G-G \cap H$. If we put $m_{x^{\circ}}=u / w$ for $x^{0} \in G \cap H$ and $m_{x^{\circ}}=0$ for $x^{0} \in G-G \cap H$, then $\mathbb{S}=\left\{\left(m_{x^{\circ}}, U\left(x^{0}\right)\right) ; x^{0} \in G\right\}$ forms an additive Cousin's distribution in $G$. Since 
$G$ is a Cousin-I domain, there exists a meromorphic function $m$ in $G$ which is a solution of (5. We put $v=w m$. For $x^{0} \in G \cap H, h=m-u / w$ is a holomorphic function in $U\left(x^{0}\right)$. Hence $v=w h+u$ is holomorphic in $U\left(x^{0}\right)$ and $v=u$ in $U\left(x^{0}\right) \cap H$. Hence $v$ is holomorphic and coincides with $u$ in $G \cap H$. Since $v$ is holomorphic in $G-G \cap H, v$ is a holomorphic function in $G$ with $v=u$ in $G \cap H$. Hence the canonical homomorphism $H^{0}(G, \mathfrak{D}) \rightarrow H^{0}(G \cap H, \mathfrak{D})$ is surjective.

LEMMA 3. Let $G$ be a domain in the space $C^{n}$ of variables $z=\left(z_{1}, z_{2}, \cdots, z_{n}\right)$. Then $G$ is a domain of holomorphy if and only if the intersection $G \cap H$ of $G$ and an l-dimensional analytic plane $H=\left\{z ; z_{\jmath}=c_{\jmath}\left(j=s_{1}, s_{2}, \cdots, s_{n-l}\right)\right\}$ is a Cousin-I open set for any integers $1 \leqq l \leqq n, 1 \leqq s_{1}<s_{2}<\cdots<s_{n-l} \leqq n$ and complex numbers $c_{\jmath}\left(j=s_{1}\right.$, $\left.s_{2}, \cdots, s_{n-l}\right)$.

Proof. Since a domain of holomorphy is a Cousin-I domain from Oka [14] and the intersection of a domain of holomorphy and an analytic plane is an open set of holomorphy, it suffices to prove the sufficiency by induction with respect to $n$. For $n=1$ any domain is a domain of holomorphy from Weierstrass' theorem. For $n=2$ any domain is a domain of holomorphy if and only if it is a Cousin-I domain from Oka [14], Cartan [5] and Behnke-Stein [2]. Suppose that our assertion is valid for $n<k(k \geqq 2)$. We consider the case $n=k$. Let $z^{0}=\left(z_{1}^{0}, z_{2}^{0}, \cdots, z_{k}^{0}\right)$ be any boundary point of $G$. Two cases (1) and (2) may occur. In the case (1) there exists $j$ such that $z^{0}$ is a boundary point of $G \cap H$ for $H=\left\{z ; z_{j}=z_{j}^{0}\right\}$. In the case (2) $z^{0}$ is not a boundary point of $G \cap H$ for $H=\left\{z ; z_{j}=z_{j}^{0}\right\}$ for any $j$.

Case (1) Since $G \cap H$ is an open set of holomorphy in $H$ from the assumption of our induction, there exists a holomorphic function $u$ in $G \cap H$ which is unbounded at $z^{0}$. From Lemma 2 there exists a holomorphic function $v$ in $G$ with $v=u$ in $G \cap H . \quad v$ is a holomorphic function in $G$ which is unbounded at $z^{0}$.

Case (2) We shall prove that there exists a sequence $\left\{z^{p} ; p=1,2,3, \cdots\right\}$ of $z^{p} \in \partial G \cap U$ such that each $z^{p}$ has the property as in the case (1) and $z^{p} \rightarrow z^{0}$ when $p \rightarrow \infty$. If this is not true, there exists $\varepsilon>0$ such that $G \cap U \cap\left\{z ; z_{\jmath}=\zeta_{\jmath}\right\}=U$ $\cap\left\{z ; z_{j}=\zeta_{\jmath}\right\}$ for $U=\left\{z ;\left|z_{j}-z_{j}^{0}\right|<\varepsilon(j=1,2, \cdots, k)\right\}$ and for any $j$ and $\zeta \in G \cap U$. Let $z^{1}=\left(z_{1}^{1}, z_{2}^{1}, \cdots, z_{k}^{1}\right)$ be any point of $G \cap U$ and $z^{2}=\left(z_{1}^{2}, z_{2}^{2}, \cdots, z_{k}^{2}\right)$ be any point of $U$. By induction we can prove that $\left(z_{1}^{2}, z_{2}^{2}, \cdots, z_{m}^{2}, z_{m+1}^{1}, \cdots, z_{k}^{1}\right) \in G \cap U$ for $1 \leqq m \leqq k$. Therefore we have $z^{2} \in G \cap U$. Hence it holds that $G \cap U=U$. This means that $z^{0}$ is an interior point of $G$. But this is a contradiction. Therefore there exists a sequence $\left\{f_{p} ; p\right.$ $=1,2,3, \cdots\}$ of holomorphic functions $f_{p}$ in $G$ which is unbounded at $z^{p}$ tending to $z^{0}$ when $p \rightarrow \infty$. From Bochner-Martin [3] there exists a holomorphic function which is unbounded at $z^{0}$.

Thus we have proved the existence of a holomorphic function in $G$ which is unbounded at $z^{0}$. Since $z^{0}$ is any boundary point of $G$, there exists a holomorphic function $f$ in $G$ which is unbounded at each boundary point of $G$ from BochnerMartin [3]. Hence $G$ is a domain of holomorphy of $f$.

Quite similarly we can prove that a domain $G$ in the space $C^{n}$ of variables $z=\left(z_{1}, z_{2}, \cdots, z_{n}\right)$ is a domain of holomorphy if and only if the canonical homomor- 
phism of $H^{0}(G, D)$ into $H^{0}(G \cap H, D)$ is surjective for any analytic plane $H$ as in Lemma 3. This is a characterization of a domain of holomorphy.

Lemma 4. If a domain $G$ in $C^{n}$ is exhausted by regular domains, then the intersection $G \cap H$ of $G$ and an l-dimensional analytic plane $H=\left\{z=\left(z_{1}, z_{2}, \cdots, z_{n}\right)\right.$; $\left.z_{j}=c_{\jmath}\left(j=s_{1}, s_{2}, \cdots, s_{n-l}\right)\right\}$ is a Cousin-I open set for any integers $1 \leqq l \leqq n, 1 \leqq s_{1}<s_{2}<$ $\cdots<s_{n-l} \leqq n$ and complex numbers $c_{\jmath}\left(j=s_{1}, s_{2}, \cdots, s_{n-l}\right)$.

Proof. There exists a sequence $\left\{G_{p} ; p=1,2,3, \cdots\right\}$ of regular domains $G_{p}$ such that $G_{p} \Subset G_{p+1}(p=1,2,3, \cdots)$ and $G=\cup_{p=1}^{\infty} G_{p}$. We may suppose that $H=\left\{(z, w)=\left(z_{1}, z_{2}\right.\right.$, $\left.\left.\cdots, z_{l}, w_{1}, w_{2}, \cdots, w_{n-l}\right) ; w_{j}=0(j=1,2, \cdots, n-l)\right\}$. There exists $\varepsilon_{p}>0$ such that $E_{p}=G_{p} \cap\left\{(z, w) ;\left|w_{j}\right|<\varepsilon_{p} ;(j=1,2, \cdots, n-l)\right\} \subset\left\{(z, w) ;\left|w_{j}\right|<\varepsilon_{p},(z, 0) \in G \cap H(j=1,2, \cdots\right.$, $n-l)\}$ for any $p$. Since $G_{p}$ is regular, $E_{p}$ is a Cousin-I open set from Lemma 1 . Let $\mathfrak{s}=\left\{\left(m_{i}, V_{i}\right) ; i \in I\right\}$ be an additive Cousin's distribution in $G \cap H$. If we put $V_{i}^{p}=G_{p} \cap\left\{(z, w) ;\left|w_{j}\right|<\varepsilon_{p},(z, 0) \in V_{\imath}(j=1,2, \cdots, n-l)\right\}$ and $M_{i}^{p}(z, w)=m_{i}(z)$ in $V_{\imath}^{p}$, then $\aleph_{p}=\left\{\left(M_{\imath}^{p}, V_{\imath}^{p}\right) ; i \in I\right\}$ is an additive Cousin's distribution in $E_{p}$. Since $E_{p}$ is a Cousin-I open set, $\mathfrak{S}_{p}$ has a solution $M^{p}(z, w)$ for any $p$. Since the set of all poles of $M^{p}(z, w)$ does not contain connected components of $G_{p} \cap H$ for any $p$, the restriction $m^{p}(z)$ of $M^{p}(z, w)$ to $G_{p} \cap H$ is a solution of the restriction $\left\{\left(m_{i} \mid G_{p} \cap H, V_{\imath} \cap G_{p}\right)\right.$;

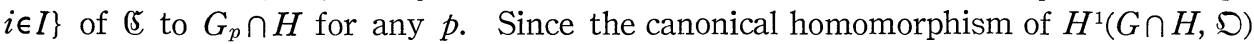
into $\lim _{p \rightarrow \infty} H^{1}\left(G_{p} \cap H, D\right)$ is injective (Lemma 6 in the previous paper [12] of the author), (5 has a solution in $G \cap H$. Therefore $G \cap H$ is a Cousin-I open set.

Proposition 1. A domain $G$ in $C^{n}$ is a domain of holomorphy if and only if it is exhausted by regular domains.

Proof. If $G$ is a domain of holomorphy, $G$ is exhausted by domains of holomorphy $G_{p}$. Since each $G_{p}$ is a regular domain, $G$ is exhausted by regular domains. Conversely, if $G$ is exhausted by regular domains, $G$ is a domain of holomorphy from Lemmas 3 and 4 .

Proposition 1 gives a characterization of a domain of holomorphy by means of Cousin-I problem and means that regular domains which are not domains of holomorphy are isolated in some sense in the set of regular domains.

\section{$\S 2$. Behaviour of a regular domain at a continuous boundary point.}

A subset $S$ of $R^{n}$ is called smooth at $x^{0} \in S$ if there exists a continuously differentiable function $f$ in a neighbourhood $U$ of $x^{0}$ such that $S \cap U=\{x ; f(x)=0$, $x \in U\}$ and $\sum_{j=1}^{n}\left(\partial f / \partial x_{j}\right)^{2}>0$ at $x^{0}$. If $\partial f / \partial x_{\jmath} \neq 0$ at $x^{0}$, there exists a continuously differentiable function $g$ in a neighbourhood $V \subset U$ of $x^{0}$ such that $S \cap V=\left\{x ; x_{3}\right.$ $\left.=g\left(x_{1}, x_{2}, \cdots, \hat{x}_{\jmath}, \cdots, x_{n}\right), x \in V\right\}$. The notion of smoothness is invariant under continuously bidifferentiable mappings. A subset $S$ of $R^{n}$ is called continuous at $x^{0} \in S$ if there exists a continuous function $g$ in a neighbourhood $V$ of $x^{0}$ such that $S \cap V$ $=\left\{x ; x_{j}=g\left(x_{1}, x_{2}, \cdots, \hat{x}_{\jmath}, \cdots, x_{n}\right), x \in V\right\}$ for some $j$. This definition may depend on 
the special choice of coordinates in $R^{n}$. A boundary point $x^{0}$ of an open set $G$ in $R^{n}$ is called continuous (or smooth) if $\partial G$ is continuous (or smooth) at $x^{0}$.

An open set $G$ in a complex manifold is called pseudoconvex at $x^{0} \in \partial G$ if there exists an open neighbourhood $V$ of $x^{0}$ such that $G \cap V$ is holomorphically convex. $G$ is called pseudoconvex if $G$ is pseudoconvex at each point of $\partial G$.

Proposition 2. A regular open set $G$ in $C^{n}$ is pseudoconvex at a continuous boundary point $z^{0}$ of $G$.

Proof. Without loss of generality we may suppose that $\partial G \cap V=\left\{z=\left(z_{1}, z_{2}, \cdots\right.\right.$, $\left.\left.z_{n}\right) ; x_{n}=g\left(z_{1}, z_{2}, \cdots, z_{n-1}, y_{n}\right), z \in V\right\}$ for a continuous function $g$ in a polycylindrical neighbourhood $V$ of $z^{0}$ where $z_{n}=x_{n}+\sqrt{-1} y_{n}$. Then two cases (1) and (2) may occur for a sufficiently small $V$. In the case (1) there holds $G \cap V=\left\{z ; x_{n}<g\left(z_{1}, z_{2}\right.\right.$, $\left.\left.\cdots, z_{n-1}, y_{n}\right), z \in V\right\}$ or $G \cap V=\left\{z ; x_{n}>g\left(z_{1}, z_{2}, \cdots, z_{n-1}, y_{n}\right), z \in V\right\}$. In the case (2) there holds $G \cap V=\left\{z ; x_{n} \neq g\left(z_{1}, z_{2}, \cdots, z_{n-1}, y_{n}\right), z \in V\right\}$.

Case (1) We have only to consider the case $G \cap V=\left\{z ; x_{n}<g\left(z_{1}, z_{2}, \cdots, z_{n-1}, y_{n}\right)\right.$, $z \in V\}$. There exists a family $\left\{V_{t} ; 0 \leqq t \leqq t_{0}\right\}$ of polycylinders $V_{t}$ containing $z^{0}$ such that $V_{t_{1}} \Subset V_{t_{2}} \Subset V$ for $0 \leqq t_{2}<t_{1} \leqq t_{0}, V_{0}=\cup_{0<t \leqq t_{0}} V_{t}$ and $\left\{z ;\left(z_{1}, z_{2}, \cdots, z_{n}-t\right) \in V_{t}\right\} \subset V$ for $0 \leqq t \leqq t_{0}$. We shall prove that $E_{t}=\left\{z ; x_{n}<g\left(z_{1}, z_{2}, \cdots, z_{n-1}, y_{n}\right)-t, z \in V_{t}\right\}$ is a regular open set for $0 \leqq t \leqq t_{0}$. Let $P$ be a polycylinder. We consider a biholomorphic mapping $\left(z_{1}, z_{2}, \cdots, z_{n}\right) \rightarrow\left(w_{1}, w_{2}, \cdots, w_{n}\right)$ defined by $w_{j}=z_{\jmath}(j=1,2, \cdots, n-1)$ and $w_{n}=z_{n}+t$. Then $E_{t} \cap P$ is mapped onto $\left\{w ; u_{n}<g\left(w_{2}, w_{2}, \cdots, w_{n-1}, v_{n}\right),\left(w_{1}, w_{2}, \cdots\right.\right.$, $\left.\left.w_{n-1}, w_{n}-t\right) \in V_{t} \cap P\right\}=G \cap V \cap\left\{z ;\left(z_{1}, z_{2}, \cdots, z_{n-1}, z_{n}-t\right) \in V_{t} \cap P\right\}$ which is a Cousin-I open set for $0 \leqq t \leqq t_{0}$ as the third element of the right-hand side of the above equation is a polycylinder. Hence $E_{t}$ is a regular open set. Since $E=G \cap V_{0}$ is exhausted by regular open sets $E_{t}, E$ is an open set of holomorphy from Puposition 1 . Hence $G$ is pseudoconvex at $z^{0}$.

Case (2) If we put $E_{1}=\left\{z ; x_{n}<g\left(z_{1}, z_{2}, \cdots, z_{n-1}, y_{n}\right), x \in V\right\}$ and $E_{2}=\left\{z ; x_{n}\right.$ $\left.>g\left(z_{1}, z_{2}, \cdots, z_{n-1}, y_{n}\right), x \in V\right\}$, then $E_{1}$ and $E_{2}$ are regular open sets. Therefore from the case (1) $E_{1}$ and $E_{2}$ are pseudoconvex at $z^{0}$. Hence $G$ is pseudoconvex at $z^{0}$.

\section{$\S 3$. Global character of locally regular domains.}

An open set $G$ in a complex manifold $M$ is called strongly regular if $G \cap D$ is a Cousin-I open set for any Stein manifold $D \subset M$. This is invariant under biholomorphic mappings of $M$. We say that a domain $G$ in a complex manifold is exhausted by strongly regular domains if there exists a sequence of strongly regular domains $G_{p}$ such that $G_{p} \Subset G_{p+1}(p=1,2,3, \cdots)$ and $G=\cup_{p=1}^{\infty} G_{p}$.

Proposition 3. A domain $G$ in a Stein manifold is a Stern manifold if and only if $G$ is exhausted by strongly regular domains.

Proof. If $G$ is a Stein manifold, it is obvious that $G$ is exhausted by strongly regular domains. Conversely suppose that $G$ is exhausted by strongly regular 
domains $G_{p}$. Let $x^{0}$ be any point of $\partial G$. There exists a biholomorphic mapping $\tau$ of a holomorphically convex neighbourhood $U$ of $x^{0}$ into a complex Euclidean space. $U$ is exhausted by holomorphically convex domains $U_{p}$. Since $\tau(G \cap U)$ is exhausted by strongly regular open sets $\tau\left(G_{p} \cap U_{p}\right), \tau(G \cap U)$ is an open set of holomorphy from Proposition 1. $G$ is a Stein manifold from Docquier-Grauert [8].

An open set $G$ in a complex manifold is called locally regular (or locally strongly regular) at a point $x^{0} \in \partial G$ if there exists a biholomorphic mapping $\tau$ of a neighbourhood $U$ of $x^{0}$ into a complex Euclidean space such that $\tau(G \cap U)$ is a regular (or strongly regular) open set. We say that $G$ is locally regular (or locally strongy regular) if $G$ is locally regular (or locally strongly regular) at each point of $\partial G$. We say that a boundary point $x^{0}$ of an open set $G$ in a differentiable manifold is a smooth boundary point of $G$ if there exists a continuously bidifferentiable mapping $\tau$ of a neighbourhood $U$ of $x^{0}$ into a Euclidean space $R^{n}$ such that $\tau\left(x^{0}\right)$ is a smooth boundary of $\tau(G \cap U)$. We say that $G$ has a smooth boundary if each point of $\partial G$ is a smooth boundary point of $G$.

Proposition 4. Let $G$ be a domain with a smooth boundary in a Stein manifold. Then $G$ is a Stern manifold if and only if $G$ is locally regular.

Proof. If $G$ is a Stein manifold, it is obvious that $G$ is locally regular. Conversely suppose that $G$ is locally regular. Let $x^{0}$ be any point of $\partial G$. Since $G$ is locally regular at $x^{0}$, there exists a biholomorphic mapping $\tau$ of a neighbourhood $U$ of $x^{0}$ into a complex Euclidean space such that $\tau(G \cap U)$ is a regular open set. Since $x^{0}$ is a smooth boundary point, there exists a continuously bidifferentiable mapping $\tau^{\prime}$ of a neighbourhood $V$ of $x^{0}$ such that $\tau^{\prime}\left(x^{0}\right)$ is a smooth boundary point of $\tau^{\prime}(G \cap V)$. Let $W$ be a polycylinder such that $\tau\left(x^{0}\right) \in W \subset \tau(U \cap V)$. Since the continuously bidifferentiable mapping $\tau \circ \tau^{\prime-1}$ maps $\tau^{\prime}\left(\tau^{-1}(W)\right)$ onto $W, \tau\left(x^{0}\right)$ is a smooth boundary point of a regular open set $\tau(G \cap U) \cap W$. From Proposition $2 \tau(G \cap U) \cap W$ is pseudoconvex at $\tau\left(x^{0}\right)$. Therefore $G$ is pseudoconvex at $x^{0}$. From DocquierGrauert [8] $G$ is a Stein manifold.

We say that a boundary point $x^{0}$ of an open set $G$ in a complex manifold is a continuous boundary point of $G$ if there exists a biholomorphic mapping $\tau$ of a neighbourhood $U$ of $x^{0}$ into a complex Euclidean space such that $\tau\left(x^{0}\right)$ is a continuous boundary point of $\tau(G \cap U)$. Moreover, if $\tau(G \cap U)$ is a regular open set simultaneously, $x^{0}$ is called a continuous and locally regular boundary point of $G$. We say that $G$ has a continuous (or continuous and locally regular) boundary if each boundary point of $G$ is a continuous (or continuous and locally regular) boundary point of $G$. These definitions are not so good that a boundary point $x^{0}$ of an open set $U$ in a complex Euclidean space $C^{n}$ may not be a continuous boundary point of $U$ even if $x^{0}$ is a continuous boundary point of $U$ which is considered as a subset of a complex manifold $C^{n}$ and that a boundary point which is continuous and which is locally regular, separately may not be continuous and locally regular.

Proposition 5. Let $G$ be a domain with a continuous boundary in a Stein manifold. Then $G$ is a Stein manifold if and only if $G$ is locally strongly regular. 
Proof. If $G$ is a Stein manifold, it is obvious that $G$ is locally strongly regular. Conversely suppose that $G$ is locally strongly regular. Let $x^{0}$ be any point of $\partial G$. Since $\partial G$ is continuous at $x^{0}$, there exists a biholomorphic mapping $\tau$ of a neighbourhood $U$ of $x^{0}$ into a complex Euclidean space such that $\tau\left(x^{0}\right)$ is a continuous boundary point of $\tau(G \cap U)$. Since $G$ is locally strongly regular at $x^{0}$, there exists a biholomorphic mapping $\tau^{\prime}$ of a neighbourhood $V$ of $x^{0}$ into a complex Euclidean space such that $\tau^{\prime}(G \cap V)$ is a strongly regular open set. Let $W$ be a holomorphically convex neighbourhood of $x^{0}$ such that $W \subset U \cap V$. Then $\tau^{\prime}(G \cap V \cap W)$ is a strongly regular open set. Since the biholomarphic mapping $\tau \circ \tau^{\prime-1}$ maps $\tau^{\prime}(G \cap V \cap W)$ onto $\tau(G \cap V \cap W), \tau(G \cap V \cap W)$ is a strongly regular open set. Therefore $\tau(G \cap V \cap W)$ is pseudoconvex at the continuous boundary point $\tau\left(x^{0}\right)$ from Proposition 2. Hence $G$ is pseudoconvex at $x^{0}$. From Docquier-Grauert [8] $G$ is a Stein manifold.

Proposition 6. A domain $G$ with a continuous and locally regular boundary in a Stein manifold is a Stein manifold.

Proof. Let $x^{0}$ be any point of $\partial G$. Since $x^{0}$ is a continuous and locally regular boundary point of $G$, there exists a biholomorphic mapping $\tau$ of a neighbourhood $U$ of $x^{0}$ into a complex Euclidean space such that $\tau\left(x^{0}\right)$ is a continuous boundary point of a regular open set $\tau(G \cap U)$. From Proposition $2 \tau(G \cap U)$ is pseudoconvex at $\tau\left(x^{0}\right)$. Hence $G$ is pseudoconvex at $x^{0}$. From Docquier-Grauert [8] $G$ is a Stein manifold.

\section{§. Example.}

Let $E$ be a relatively compact open subset with a smooth boundary in a Stein manifold $M$. Then from Andreotti-Grauert [1] and Fujimoto-Kasahara [9] the canonical homomorphism $H^{0}(M, \mathfrak{D}) \rightarrow H^{0}(M-\bar{E}, \mathfrak{D})$ is surjective. Therefore $M-\bar{E}$ is not holomorphically convex. Therefore from Proposition $4, M-\bar{E}$ is not locally regular at some point of $\partial E$. Let $x^{0}$ be a point of $\partial E$ at which $M-\bar{E}$ is not locally regular. For any neighbourhood $U$ of $x^{0}$, there exists a holomorphically convex subdomain $D$ of $U$ such that $(M-\bar{E}) \cap D$ is not a Cousin-I open set. Making use of Andreotti-Grauert [1], we can take $E$ such that $M-\bar{E}$ is a Cousin-I domain. This gives an example of a Cousin-I domain with a smooth boundary which is not locally regular.

Proposition 7. Let $E$ be a relatively compact open subset of a Stein manifold $M$. Then there exists an arbitrarily small holomorphically convex subdomain $D$ of $M$ such that $(M-\bar{E}) \cap D$ is not a Cousin-I open set.

\section{References}

11] Andreotti, A., Et H. Grauert, Théorie de finitude pour la cohomologie des espaces complexes. Bull. Soc. Math. France 90 (1962), 193-260.

L2 ] BehnKe, H., Und K. Stein, Analytische Funktionen mehrerer Veränderlichen zu 
vorgegebenen Null- und Pol-stellenflächen. Jber. D. M. V. 47 (1937), 177-192.

[3] Bochner, S., AND W. T. MArtin, Several complex variables. Princeton Univ. Press (1948).

[4] Bremermann, H. J., Über die Äquivalenz der pseudokonvexen Gebiete und der Holomorphiegebiete im Raum von $n$ komplexen Veränderlichen. Math. Ann. 128 (1954), 36-91.

[5] Cartan, H., Les problèmes de Poincaré et de Cousin pour les fonctions de plusieurs variables complexes. C. R. Paris 198 (1934), 1284-1287.

[6] Cartan, H., Sur les premières problèmes de Cousin. ibid. 207 (1938), 558-560.

[7] Cartan. H., und P. Thullen, Zur Theorie der Singularitäten der Funktionen mehrerer komplexen Veränderlichen: Regularitäts- und Konvergezbereiche. Math. Ann. 106 (1932), 617-647.

[8] Docquier, F., und H. Grauert, Levisches Problem und Rungescher Satz für Teilgebiete Steinscher Mannigfaltigkeit. Math. Ann. 140 (1960), 94-123.

[9] Fujimoto, H., AND K. Kasahara, On the continuability of holomorphic functions on complex manifolds. To appear in J. Math. Soc. Japan 16 (1964).

[10] Hitotumatu, S., A note on Levi's conjecture. Comm. Math. Univ. Sancti Pauli 4 (1955), 105-108.

[11] Kajrwara, J., Note on the Levi problem. Sci. Rep. Kanazawa Univ. 8 (1963), 259-270.

[12] KaJiwara, J., On the limit of a monotonous sequence of Cousin's domains. To appear in J. Math. Soc. Japan.

[13] Norguet, F.. Sur les domaines d'holomorphies des fonctions uniformes de plusieurs variables complexes (Passage du local au global). Bull. Soc. Math. France 82 (1954), 137-159.

[14] Ока, K., Sur les fonctions analytiques de plusieurs variables: II Domaines d'holomorphie. J. Sci. Hiroshima Univ. 7 (1937), 115-130.

[15] ОкА, K., Sur les fonctions analytiques de plusieurs variables: IX Domaines finis sans point critique intérieur. Jap. J. Math. 23 (1953). 97-155.

[16] ScheJA, G., Riemannsche Hebbarkeitssätze für Cohomologieklassen. Math. Ann. 144 (1961). 345-360.

Department of Mathematics, Kanazawa University. 